\title{
Towards Washable Electrotextile UHF RFID Tags: Reliability Study of Epoxy-Coated Copper Fabric Antennas
}

\author{
Shiqi Wang, ${ }^{1}$ Ngai Lok Chong, ${ }^{1}$ Johanna Virkki, ${ }^{2}$ Toni Björninen, \\ Lauri Sydänheimo, ${ }^{2}$ and Leena Ukkonen ${ }^{2}$ \\ ${ }^{1}$ Department of Electronic Engineering, City University of Hong Kong, 83 Tat Chee Avenue, Kowloon, Hong Kong \\ ${ }^{2}$ Department of Electronics and Communications Engineering, Tampere University of Technology, 33720 Tampere, Finland
}

Correspondence should be addressed to Johanna Virkki; johanna.virkki@tut.fi

Received 16 October 2015; Accepted 8 November 2015

Academic Editor: Luciano Tarricone

Copyright (C) 2015 Shiqi Wang et al. This is an open access article distributed under the Creative Commons Attribution License, which permits unrestricted use, distribution, and reproduction in any medium, provided the original work is properly cited.

We investigate the impact of washing on the performance of passive UHF RFID tags based on dipole antennas fabricated from copper fabric and coated with protective epoxy coating. Initially, the tags achieved read ranges of about 8 meters, under the European RFID emission regulation. To assess the impact of washing on the performance of the tags, they were washed repeatedly in a washing machine and measured after every washing cycle. Despite the reliability challenges related to mechanical stress, the used epoxy coating was found to be a promising coating for electrotextile tags in moist conditions.

\section{Introduction}

Wireless body area networks are an increasingly important area of research and the future expectations on wearable electronics are high [1-3]. For example, wearable RFID (radiofrequency identification) tags have enormous potential in future monitoring, welfare, and healthcare applications. In this study, antennas for wearable passive ultra-high-frequency (UHF) RFID tags were fabricated from commercially available copper fabric and coated with a protective epoxy layer. To evaluate the reliability of the tags, they were washed repeatedly in a household washing machine using a common $40^{\circ} \mathrm{C}$ washing program with detergent and spinning and tested wirelessly after each washing cycle. All the tags were washed at the same time in a single washing bag with no other laundry. The impact of washing on noncoated copper fabric was also tested by washing a sheet of copper fabric without any coating and using the washed fabric for the fabricating of tags.

The remainder of the text is organized as follows: Section 2 gives a short review of UHF RFID systems and shortly introduces the development of wearable antennas and RFID tags. Section 3 introduces our tag design and the fabrication of tags as well as the brush-painting of the protective coating. The tag measurements are presented in Section 4. Section 5 presents and discusses the measurement results before and after washing and analyzes the tag failures. Section 6 summarizes the conclusions of this study.

\section{Textile Antennas and RFID Tags}

Passive RFID technology provides the automatic identification and tracking of items. This is achieved with batteryfree remotely addressable electronic tags composed of an antenna and an integrated circuit (IC). The use of propagating electromagnetic waves in the UHF frequency range for powering and communicating with the passive tags enables rapid interrogation of a large number of tags through various media. In comparison to barcodes and chipless RFID tags, IC-enabled tags allow the data stored in them to be updated wirelessly at any time. Thanks to the energy-efficient mechanism of digitally modulated scattering utilized in the tag-toreader wireless communication, the data can be read from the distance of several meters and specialized antennas can provide reading distances reaching 25 meters without an onboard energy source. This makes the battery-free passive tags promising candidates as sensing platforms and digital entities in Internet of Things [4]. 
For further information on RFID technology, we refer to landmark articles $[5,6]$ presenting early investigations and [7] which provides a comprehensive introduction to today's passive UHF RFID systems. A thorough discussion on antennas for RFID tags can be found in [8]. Until today, new applications based on the versatile RFID technology are continually emerging. In particular, the passive tags are promising candidates to provide energy-autonomous wireless sensors, which exhibit low complexity and cost $[2,4$, $9,10]$. This makes them a promising technology for body area networks. Overall, this development is building upon and extending the great amount of research on wearable antennas conducted during the past decade. It commenced with the introduction of fully textile-based antennas for smart clothing [11-14] and was followed by numerous application examples, including FM radio reception [15], protective clothing for firefighters [16], and space suits [17].

Simultaneously, the high-frequency characterization of novel electrotextile materials provided insight on the attainable electromagnetic performance. Early investigations included electromagnetic characterization of transmission lines formed by embedding of metallic yearns into fabrics [18] and by screen printing of inks with metallic inclusions [19]. Further assessment on screen printing and investigation of stretchable screen printed conductors under tensile load was provided in articles $[20,21]$. Characterization of electrotextiles based on conductive threads and metal coated fabrics specifically for wearable antennas was further discussed in articles [22, 23]. A look at the current state-of-the-art of embroidery and related manufacturing techniques is presented in publication [24].

Today, embroidery and metallized fabrics are commonly reported methods of creating antennas for wearable passive UHF RFID tags [9, 25-27]. Progress has also been made in inkjet-printing fabrication of antennas based on metal nanoparticle inks on leather [28] and fabrics [29-31]. RFID tag assembly based on direct write dispensing of screen printable silver and copper inks on textiles have also been presented $[32,33]$.

In addition to the assessment of the fitting mass manufacturing processes and materials, the robustness of textile antennas toward various environmental stresses, such as exposure to extreme climatic conditions [34] and mechanical stress, including stretching [35], crumpling [36], and bending $[14,37,38]$, as well as absorption of moisture [39-41], is another major issue to be addressed before the large-scale deployment of body-worn antennas and RFID tags. Finally, machine washing [40, 42-46] has been established detrimental to the electrical performance of electrotextile devices, even with a limited number of wash repetitions. The challenge remains in identifying low-cost conformal coating materials that are compatible with textile materials and also meet the requirements of extremely low electromagnetic energy dissipation and mechanical durability. It should be noted that some commercial UHF RFID laundry tags are already available on the marked, for example, Fujitsu WT-A522 UHF RFID Laundry Tag [47] and TAGSYS' LinTRAK UHF Tag [48].

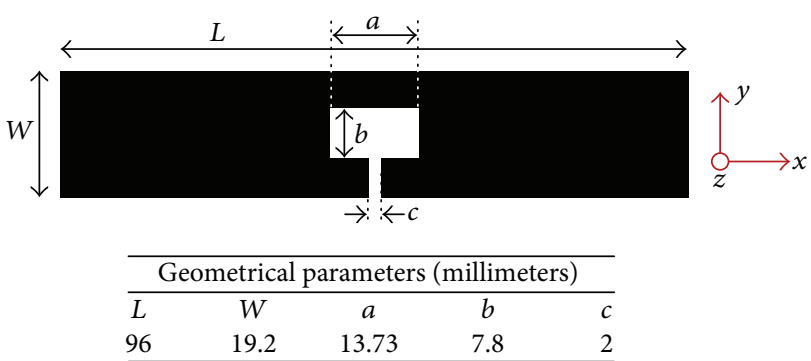

Figure 1: Antenna geometry.

\section{Fabrication and Coating of Tags}

We chose the tags antennas to be T-matched dipoles with the structure shown in Figure 1. This a very common class of antennas used in UHF RFID tags. In this work, the length (L) of the antenna was chosen to be $96 \mathrm{~mm}$, which is similar to many commercial products and sufficient to avoid the ailments of electrically small antennas in the considered frequencies from $800 \mathrm{MHz}$ to $1 \mathrm{GHz}$. The tag antennas were patterned from Less EMF Pure Copper Polyester Taffeta Fabric (Cat. \#A1212) [49], which is a thin and lightweight fabric and can be easily cut with scissors. The sheet resistance of the fabric has been estimated as $0.4 \Omega / \mathrm{Sq}$. The tag IC utilized in this experiment was NXP UCODE G2iL series IC [50], which has a low wake-up power of $15.8 \mu \mathrm{W}(-18 \mathrm{dBm})$.

We modelled the tag IC and the copper fixture as a parallel connection of a resistance of $2.85 \mathrm{k} \Omega$ and capacitance of $0.91 \mathrm{pF}$, which represents the IC input at the wake-up power of the circuit [9]. In this work, we studied an antenna that achieved the complex-conjugate impedance matching with the chip around $970 \mathrm{MHz}$. This is somewhat higher than, for example, the centre frequencies of $866.5 \mathrm{MHz}$ and $915 \mathrm{MHz}$ specified for UHF RFID systems in Europe and North America, respectively, but it was chosen since in the future work these tags will be studied in the body-worn configuration where significant downward shift in operation frequency is expected due to the proximity of human body with high dielectric constant.

Normally, the read range of passive tags is limited by the forward link operation, that is, the efficiency of the wireless power transfer from the reader to the tag IC. Assuming freespace conditions for site-independent comparison, the attainable read range of the tag in the directions $\phi$ and $\theta$ of a spherical coordinate system with the tag in its origin is given by [8]

$$
\begin{array}{r}
d_{\mathrm{tag}}(\phi, \theta)=\frac{\lambda}{4 \pi} \sqrt{\frac{\chi_{\mathrm{pol}}(\phi, \theta) \tau e_{r} D(\phi, \theta) \mathrm{EIRP}}{P_{\mathrm{ic} 0}}} ; \\
\tau=\frac{4 \operatorname{Re}\left(Z_{\mathrm{ant}}\right) \operatorname{Re}\left(Z_{\mathrm{ic}}\right)}{\left|Z_{\mathrm{ant}}+Z_{\mathrm{ic}}\right|^{2}},
\end{array}
$$

where $\lambda$ is the wavelength of the reader's carrier signal, EIRP is the regulated equivalent isotropic radiated power of the reader, $P_{\mathrm{ico}}$ is the wake-up power of the tag IC, $\chi_{\mathrm{pol}}$ is the mutual polarization power efficiency between the tag and reader antennas, $e_{r}$ is the tag antenna radiation efficiency, 


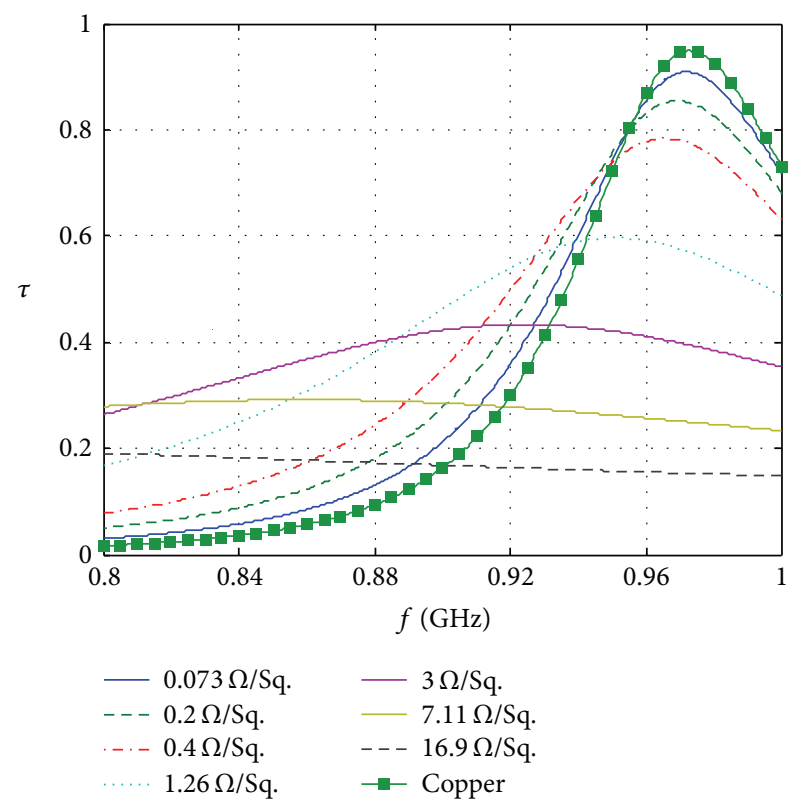

Figure 2: Simulated antenna-IC power transfer efficiency.

$D$ is the tag antenna directivity, and $\tau$ is the antenna-IC power transfer efficiency determined by the antenna and IC impedances $Z_{\text {ant }}$ and $Z_{\text {ic }}$, respectively. Equation (1) is a direct implication of Friis' simple transmission equation. Below we will report $d_{\text {tag }}$ under the European RFID emission regulation: $\mathrm{EIRP}=3.28 \mathrm{~W}$, assuming aligned reader and tag antenna polarizations: $\chi_{\text {pol }}=1$.

To provide insight on the nominal electromagnetic performance of the textile tag and to predict the impact of reduction in the antenna conductivity, we simulated the antenna using ANSYS HFSS version 15 (full-wave electromagnetic solver based on finite element method) with various sheet resistance values assigned for the conductor. The results, including a comparison with a tag based on an antenna made of $20 \mu \mathrm{m}$ thick sheet of bulk copper, are presented in Figures $2-4$.

The results show that, for low values of sheet resistance, $\tau$ peaks approximately at $970 \mathrm{MHz}$. For larger values, the peak disappears and the antenna becomes detuned. In the extreme case of $16.9 \Omega / \mathrm{Sq}$, less than $20 \%$ of the power captured by the antenna is delivered to the IC. This was observed to be mainly due to the increase in antenna resistance, but also due to the decrease in antenna reactance, although it became notable only for large values of sheet resistance, $7.11 \Omega / \mathrm{Sq}$ and beyond. Figure 3 shows the impact of sheet resistance on the antenna radiation efficiency. Expectedly, it decreases with an increasing sheet resistance. However, at $970 \mathrm{MHz}$, the nominal sheet resistance of $0.4 \Omega / \mathrm{Sq}$ is still expected to provide $e_{r}$ of more than $50 \%$. From Figure 4, we can see that this performance translates to attainable read range of 8.5 meters at $970 \mathrm{MHz}$. In comparison to the tag with a bulk copper antenna, this is 4.2 meters less, but without a doubt sufficient for many applications of textile-based wearable systems.

The manufacturer had mounted the chip on a fixture with two $3 \times 3 \mathrm{~mm}^{2}$ pads which we connected to the antenna

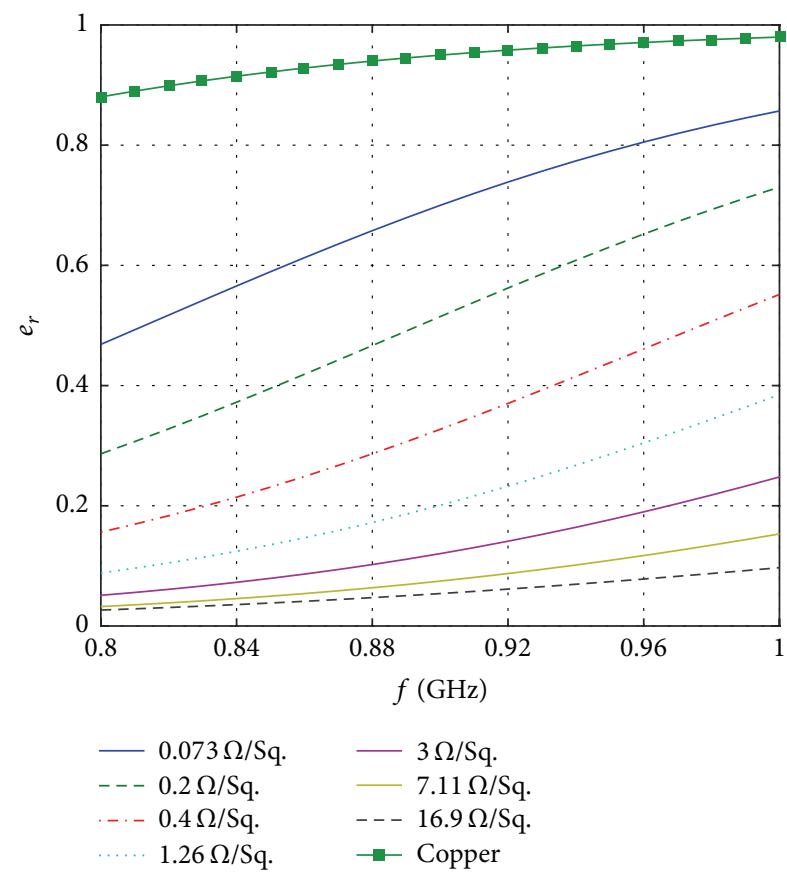

FIGURE 3: Simulated antenna radiation efficiency.

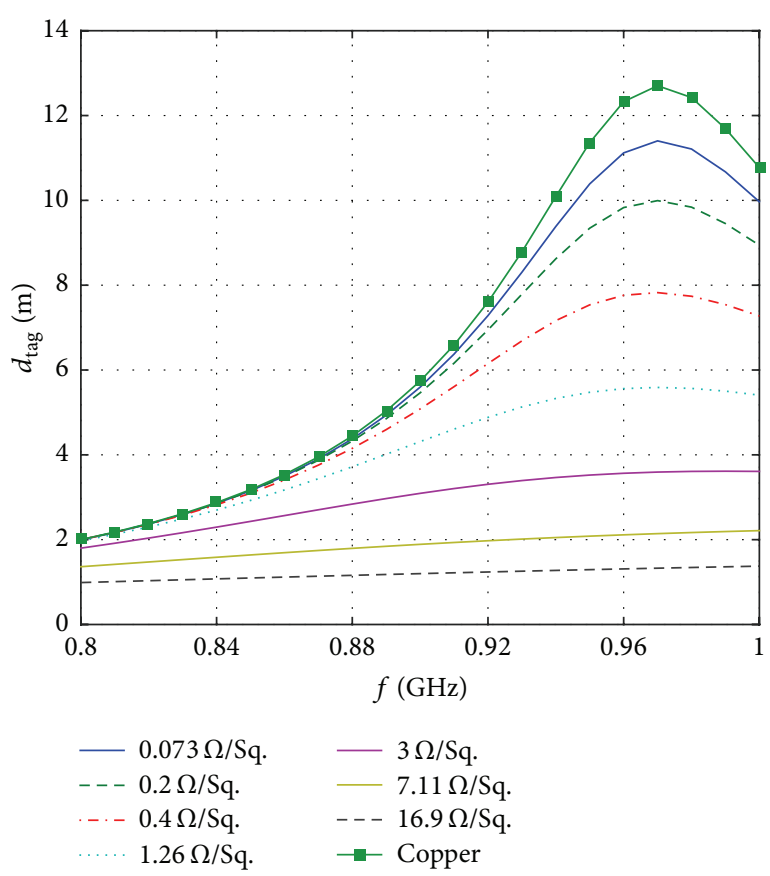

FIGURE 4: Simulated attainable read range of the tag.

terminals using conductive epoxy (Circuit Works CW2400). From our previous study, we knew that the copper electrotextile tags do not endure washing without a protective coating, as the noncoated copper fabric tag became dysfunctional after four wash repetitions in a study published in [42]. Thus, ESL 243 White epoxy coating [51] was applied by brushpainting it to cover the tags completely. The tags were coated on one side and dried for 40 minutes in $115^{\circ} \mathrm{C}$, which was then 


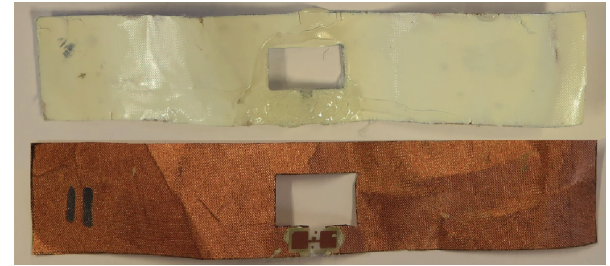

FIGURE 5: Noncoated (lower) and coated (upper) copper fabric tags.

repeated for the other side, so that the coating covered the whole tag. The long drying time and low drying temperature (recommendation from the coating manufacturer: drying in $125^{\circ} \mathrm{C}$ for $10-15$ minutes and curing in $150^{\circ} \mathrm{C}$ for $5-15$ minutes) were chosen because of the low temperature endurance of the tag IC. Only one layer of coating was used. After the drying of the coating, we shielded the area around the IC, using regular textile glue on top of the actual protective coating, in order to protect the IC and the IC-antenna joint from the mechanical stress during washing. Figure 5 shows a coated and a noncoated tag.

\section{Wireless Measurements}

We analyzed the performance of the assembled textile RFID tags based on the measured threshold power $\left(P_{\text {th }}\right)$, which is the minimum output power of an RFID reader to activate the tag under test from a given distance. It can be measured using RFID readers and testers with adjustable output power. In this work, we have used Voyantic Tagformance measurement system [52] to conduct the measurement through a range of frequencies from $800 \mathrm{MHz}$ to $1000 \mathrm{MHz}$. Because the measured threshold power depends on the measurement site and hardware, we used the attainable free-space read range of the tag $\left(d_{\text {tag }}\right)$ derived from $P_{\text {th }}$ to provide universal tag characterization. As detailed in article [35], we can estimate $d_{\text {tag }}$ based on the measured threshold power of the tag under test and a system reference tag as

$$
d_{\mathrm{tag}}=\frac{\lambda}{4 \pi} \sqrt{\frac{\mathrm{EIRP}}{\Lambda} \frac{P_{\mathrm{th}^{*}}}{P_{\mathrm{th}}}},
$$

where $\Lambda$ is a parameter (unit: watts) describing the sensitivity of the reference tag of the measurement system and $P_{\mathrm{th}^{*}}$ is the measured threshold power of the reference tag. In this paper, we report all the read range results under the European RFID emission regulation: EIRP $=3.28 \mathrm{~W}$ in the direction of the positive $y$-axis in Figure 1 . In general, the $y z$-plane is the omnidirectional plane of the dipole antenna where the read range is approximately equal in all directions. In all measurements, the dipole tag was aligned for polarization matching with a linearly polarized reader antenna. Based on the calibration data provided by the manufacturer of the measurement system, we have estimated that the maximum variability in $d_{\text {tag }}$ due to variability in the system reference tag $(\Lambda)$ and the output power meter of the reader $\left(P_{\mathrm{th}}\right.$ and $\left.P_{\mathrm{th}}{ }^{*}\right)$ is less than $5 \%$ throughout the studied frequency range.

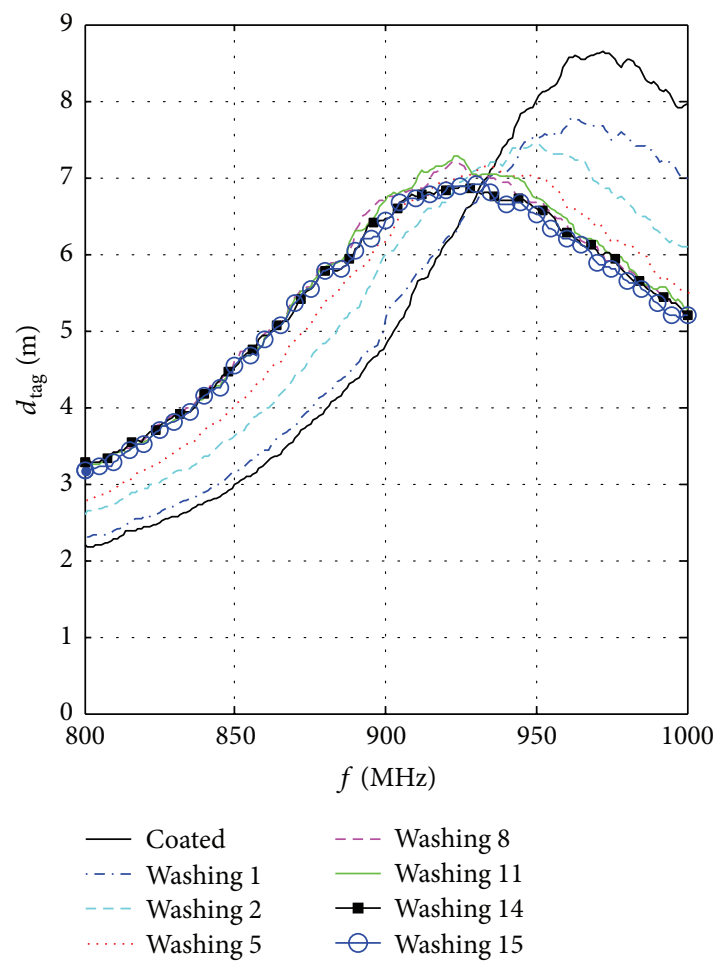

FIGURE 6: Measurement results for a tag that is working well after 15 washing cycles.

\section{Results and Discussion}

The attainable read ranges based on threshold power measurement of coated copper electrotextile tags before and after washing cycles, in the frequency range of $800-1000 \mathrm{MHz}$, are presented in Figures 6-8. The tags were washed up to 15 times or until the read range had reduced below one meter, which was considered as a failure limit in our experiment. The results show that initially the epoxy-coated copper fabric tags achieved attainable peak read ranges of approximately 7-8.5 meters, within the frequency range from $960 \mathrm{MHz}$ to $970 \mathrm{MHz}$.

It was noticed that the epoxy coating was well suited for shielding the tag from moisture and detergent. As can be seen in Figure 6, the peak read ranges after 15 washing cycles were still nearly 7 meters. The frequency of the peak read range had shifted to the frequency range $920-940 \mathrm{MHz}$, but as seen from Figure 6, this happened already during the first 5 washing cycles and no further frequency-shift or decrease in the read range was observed after that.

However, it was discovered that the mechanical stress during washing can seriously affect the tags' performance. Some tags stopped working after the first washing cycle, even though they worked fine after the coating. After studying the broken tags, it was found that the IC fixture pads remained well attached to the antenna, but the IC itself had been ripped off and remained attached to the coating material. The IC fixture of a broken tag is shown in Figure 9. The IC was most likely attached to the coating when it was brushed over the IC fixture and dried and was ripped off from the fixture 


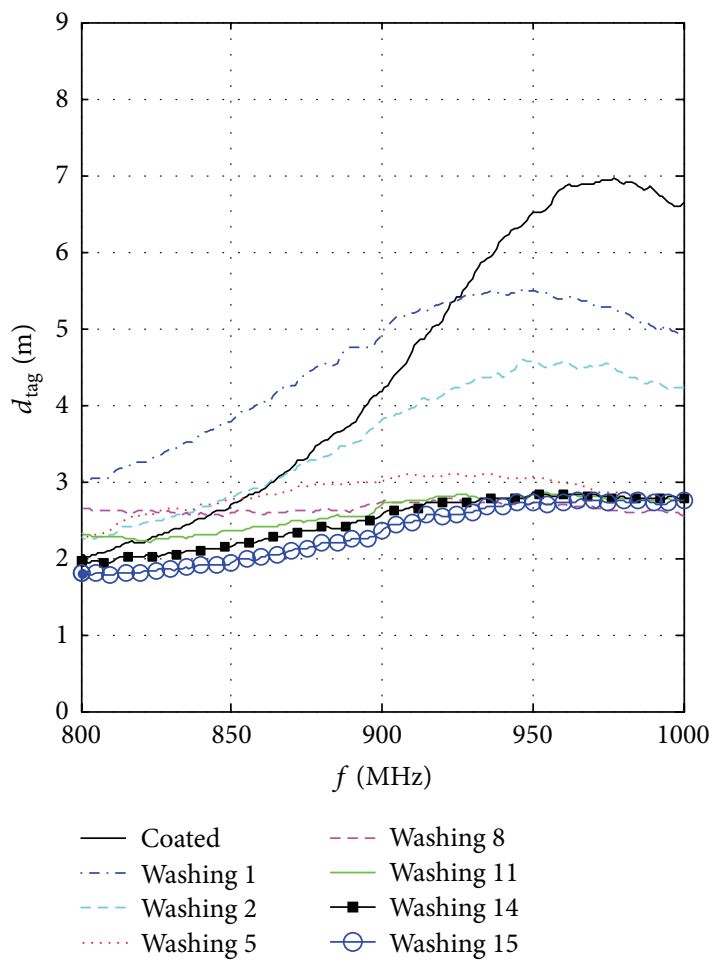

Figure 7: Measurement results for a tag where the read range was steadily decreasing after each washing cycle.

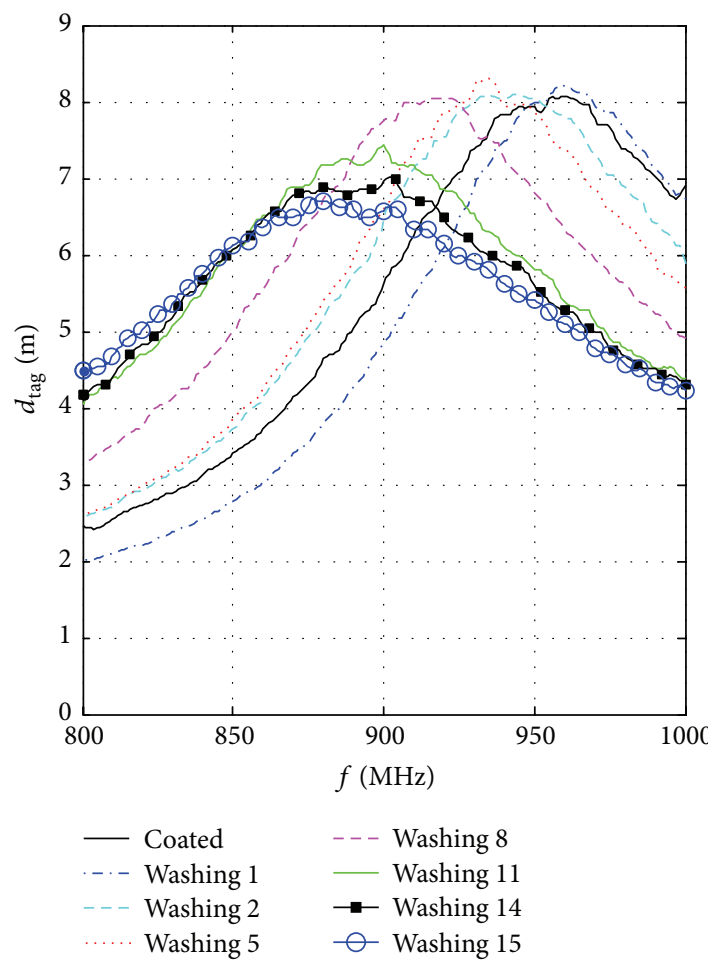

FIGURE 8: Measurement results for a tag where the peak read range was found to strongly shift to lower frequencies after each washing cycle.

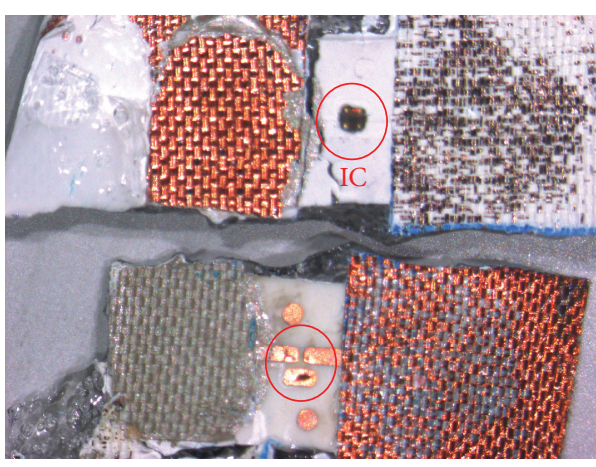

Figure 9: The IC fixture of a broken tag: the IC has been ripped off from the IC fixture and attached to the coating material; magnification is $\times 6.3$.

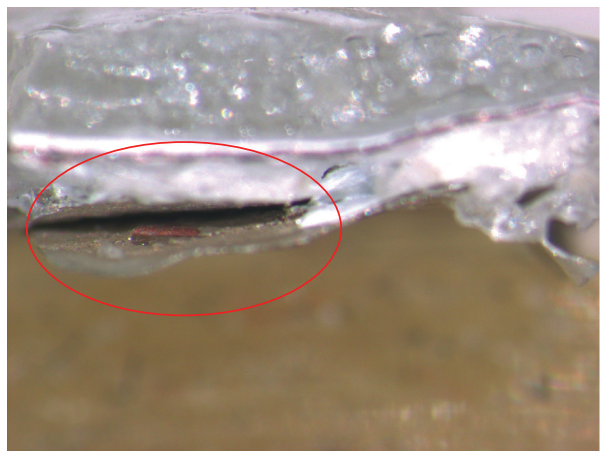

Figure 10: The attachment of the IC fixture pad to the antenna is open; magnification is $\times 20$.

because of the mechanical stress during washing, due to a stronger adhesion strength between the IC and the coating in comparison with the IC and the IC fixture. Thus, in order to protect the IC and IC-antenna joint from the mechanical stress during washing, instead of shielding the IC part with regular textile glue on top of the actual coating, the IC and the IC-antenna joint should be shielded with a flexible material already before applying the actual coating.

For all tags, a downward shift in the frequency of the peak read range was observed after 3-5 washing cycles. After this, the read range of some tags started to steadily decrease after each washing cycle (see Figure 7). For other tags, the peak read range was found to not only slightly decrease, to about 7 meters, but to also strongly shift to lower frequencies after each washing cycle. This is seen in Figure 8, where after 15 washing cycles the frequency of the peak read range was between $880 \mathrm{MHz}$ and $900 \mathrm{MHz}$. This shift in frequency and decrease in the read range may be an indication of gradual detachment of the IC. This was confirmed in our failure analysis. As shown in Figure 10, the IC fixture pad was found to be poorly attached to the antenna, most probably because of the strong mechanical stress during washing and spinning. Another reason behind the decreased read range can also be the cracking of the protective coating, again caused by mechanical stresses and deformation of the tag during washing. Examples of these cracks can be 


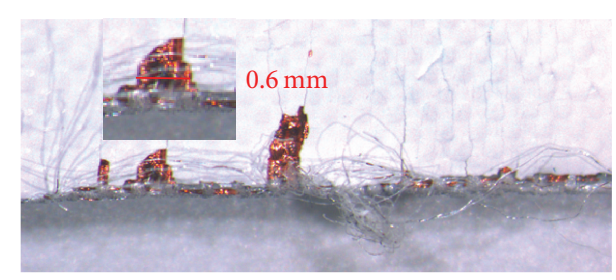

(a)

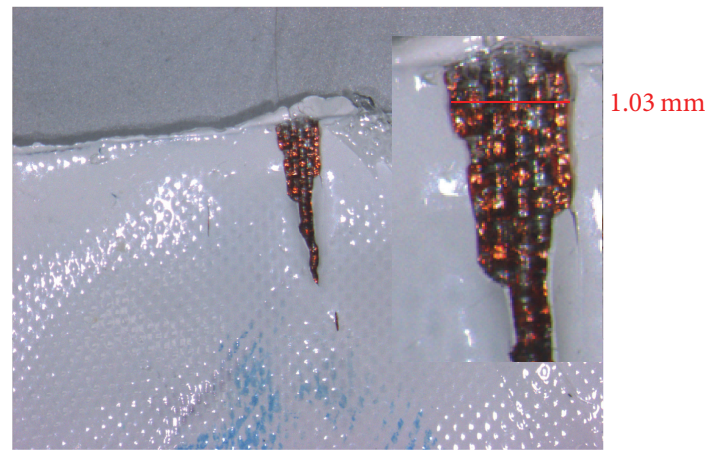

(b)

FIGURE 11: Cracks in the protective coating; magnification of the tags is $\times 12.5$.

seen in Figure 11. Even though the coating is protecting the antenna from moisture, these stress-related cracks allow the moisture to penetrate the antenna under the coating, causing a detrimental impact on the tag performance.

The impact of washing on noncoated copper fabric was also tested by washing a piece of fabric 15 times without any coating and using the washed fabric for fabricating tags. None of the fabricated tags worked, which supports the results of our earlier study [42], where it was noticed that the copper fabric is not washable without a protective coating. The epoxy coating used in this study protects textile tags from water and detergent but does not provide sufficient protection against the strong mechanical stress from washing and spinning.

Some initial washing tests for electrotextile RFID tags with acrylic coating, some epoxy coatings, textile glue, and also some silicone materials have been published [42, 43]. In our future research, besides epoxy materials, potential coating materials also include different types of silicone materials, and DuPont's PE772 [53], stretchable protective encapsulant for wearables. After these preliminary washing tests, conditions defined in standardized tests for textiles for domestic uses will be addressed [54]. In addition, on-body measurements and optimization of the antenna to be used near the body are important future topics.

\section{Conclusions}

Textile antennas enable seamlessly integrated wearable RFID tags and other wireless devices in body area networks, where new applications are continually emerging. We studied the impact of washing on epoxy-coated copper fabric textile UHF RFID tags. Tag samples were washed in a household washing machine, in a $40^{\circ} \mathrm{C}$ washing program with detergent and spinning, and tested wirelessly after each washing cycle.

Initially the epoxy-coated tags achieved attainable peak read ranges of approximately 8 meters. The tags maintained read ranges of 7 meters after 15 wash repetitions, which is very promising for future wearable applications. However, strong mechanical stress during washing and spinning was found to cause a major damaging impact on tag performance. In addition, the IC needs to be shielded with a flexible coating, which does not attach to the IC, before applying the actual coating.

\section{Conflict of Interests}

The authors declare that there is no conflict of interests regarding the publication of this paper.

\section{Acknowledgments}

This research was funded and supported by Emil Aaltonen Foundation, the Academy of Finland, City University of Hong Kong Overseas Internship Scheme 2015, Tekes, Jane and Aatos Erkko Foundation, and the European Commission Marie Curie IRSES AdvIOT.

\section{References}

[1] H.-J. Yoo, "Your heart on your sleeve: advances in textile-based electronics are weaving computers right into the clothes we wear," IEEE Solid-State Circuits Magazine, vol. 5, no. 1, pp. 5970, 2013.

[2] S. Rao, N. Llombart, E. Moradi et al., "Miniature implantable and wearable on-body antennas: towards the new era of wireless body-centric systems," IEEE Antennas and Propagation Magazine, vol. 56, no. 1, pp. 271-291, 2014.

[3] S. Lemey, F. Declercq, and H. Rogier, "Textile antennas as hybrid energy-harvesting platforms," Proceedings of the IEEE, vol. 102, no. 11, pp. 1833-1857, 2014.

[4] G. Marrocco, "Pervasive electromagnetics: sensing paradigms by passive RFID technology," IEEE Wireless Communications, vol. 17, no. 6, pp. 10-17, 2010.

[5] H. Stockman, "Communication by means of reflected power," Proceedings of the IRE, vol. 36, no. 10, pp. 1196-1204, 1948.

[6] A. R. Koelle, S. W. Depp, and R. W. Freyman, "Short-range radio-telemetry for electronic identification, using modulated RF backscatter," Proceedings of the IEEE, vol. 63, no. 8, pp. 12601261, 1975.

[7] D. Dobkin, The RF in RFID: Passive UHF RFID in Practice, Newnes-Elsevier, 2008.

[8] G. Marrocco, "The art of UHF RFID antenna design: impedance-matching and size-reduction techniques," IEEE Antennas and Propagation Magazine, vol. 50, no. 1, pp. 66-79, 2008.

[9] F. Long, X. D. Zhang, T. Björninen et al., "Implementation and wireless readout of passive UHF RFID strain sensor tags based on electro-textile antennas," in Proceedings of the 9th European Conference on Antennas and Propagation (EuCAP '15), pp. 1-5, IEEE, Lisbon, Portugal, April 2015.

[10] L. Catarinucci, R. Colella, D. De Donno, and L. Tarricone, "Fully-passive devices for RFID smart sensing," in Proceedings 
of the IEEE Antennas and Propagation Society International Symposium (APSURSI '13), pp. 2311-2312, IEEE, Orlando, Fla, USA, July 2013.

[11] P. Salonen and L. Hurme, "A novel fabric WLAN antenna for wearable applications," in Proceedings of the IEEE International Symposium on Antennas and Propagation, pp. 700-703, Columbus, Ohio, USA, June 2003.

[12] I. Locher, M. Klemm, T. Kirstein, and G. Tröster, "Design and characterization of purely textile patch antennas," IEEE Transactions on Advanced Packaging, vol. 29, no. 4, pp. 777-788, 2006.

[13] J. C. G. Matthews and G. Pettitt, "Development of flexible, wearable antennas," in Proceedings of the 3rd European Conference on Antennas and Propagation (EuCAP '09), pp. 273-277, IEEE, Berlin, Germany, March 2009.

[14] S. Zhu and R. Langley, "Dual-band wearable textile antenna on an EBG substrate," IEEE Transactions on Antennas and Propagation, vol. 57, no. 4, pp. 926-935, 2009.

[15] J.-S. Roh, Y.-S. Chi, J.-H. Lee, Y. Tak, S. Nam, and T. J. Kang, "Embroidered wearable multiresonant folded dipole antenna for FM reception," IEEE Antennas and Wireless Propagation Letters, vol. 9, pp. 803-806, 2010.

[16] C. Hertleer, H. Rogier, L. Vallozzi, and L. Van Langenhove, "A textile antenna for off-body communication integrated into protective clothing for firefighters," IEEE Transactions on Antennas and Propagation, vol. 57, no. 4, pp. 919-925, 2009.

[17] T. F. Kennedy, P. W. Fink, A. W. Chu, N. J. Champagne II, G. Y. Lin, and M. A. Khayat, "Body-worn E-textile antennas: the good, the low-mass, and the conformal," IEEE Transactions on Antennas and Propagation, vol. 57, no. 4, pp. 910-918, 2009.

[18] D. Cottet, J. Grzyb, T. Kirstein, and G. Tröster, "Electrical characterization of textile transmission lines," IEEE Transactions on Advanced Packaging, vol. 26, no. 2, pp. 182-190, 2003.

[19] I. Locher and G. Tröster, "Screen-printed textile transmission lines," Textile Research Journal, vol. 77, no. 11, pp. 837-842, 2007.

[20] S. Merilampi, T. Laine-Ma, and P. Ruuskanen, "The characterization of electrically conductive silver ink patterns on flexible substrates," Microelectronics Reliability, vol. 49, no. 7, pp. 782790, 2009.

[21] S. Merilampi, T. Björninen, V. Haukka, P. Ruuskanen, L. Ukkonen, and L. Sydänheimo, "Analysis of electrically conductive silver ink on stretchable substrates under tensile load," Microelectronics Reliability, vol. 50, no. 12, pp. 2001-2011, 2010.

[22] Y. Ouyang and W. J. Chappell, "High frequency properties of electro-textiles for wearable antenna applications," IEEE Transactions on Antennas and Propagation, vol. 56, no. 2, pp. 381-389, 2008.

[23] K. Koski, E. Moradi, A. Vena et al., "Characterization of electrotextiles using wireless reflectometry for optimization of wearable UHF RFID tags," in Proceedings of the Progress in Electromagnetics Research Symposium (PIERS '13), pp. 1188-1192, Stockholm, Sweden, August 2013.

[24] A. Tsolis, W. G. Whittow, A. Alexandridis, and J. Vardaxoglou, "Embroidery and related manufacturing techniques for wearable antennas: challenges and opportunities," Electronics, vol. 3, no. 2, pp. 314-338, 2014.

[25] Y. Kim, K. Lee, Y. Kim, and Y. Chung, "Wearable UHF RFID tag antenna design using flexible electro-thread and textile," in Proceedings of the IEEE Antennas and Propagation Society International Symposium, pp. 5487-5490, IEEE, Honolulu, Hawaii, USA, June 2007.
[26] J. H. Choi, Y. Kim, K. Lee, and Y. Chung, "Various wearable embroidery RFID tag antenna using electro-thread," in Proceedings of the IEEE Antennas and Propagation Society International Symposium, pp. 1-4, IEEE, San Diego, Calif, USA, July 2008.

[27] G. Kim, J. Lee, K. H. Lee et al., "Design of a UHF RFID fiber tag antenna with electric-thread using a sewing machine," in Proceedings of the Asia-Pacific Microwave Conference (APMC '08), pp. 1-4, IEEE, Macau, China, December 2008.

[28] M. F. Farooqui and A. Shamim, "Dual band inkjet printed bowtie slot antenna on leather," in Proceedings of the 7th European Conference on Antennas and Propagation (EuCAP '13), pp. 32873290, IEEE, Gothenburg, Sweden, April 2013.

[29] J. Lilja, V. Pynttäri, T. Kaija et al., "Body-worn antennas making a splash: lifejacket-integrated antennas for global search and rescue satellite system," IEEE Antennas and Propagation Magazine, vol. 55, no. 2, pp. 324-341, 2013.

[30] A. Chauraya, W. G. Whittow, Y. C. Vardaxoglou et al., "Inkjet printed dipole antennas on textiles for wearable communications," IET Microwaves, Antennas and Propagation, vol. 7, no. 9, pp. 760-767, 2013.

[31] W. G. Whittow, A. Chauraya, J. C. Vardaxoglou et al., "Inkjetprinted microstrip patch antennas realized on textile for wearable applications," IEEE Antennas and Wireless Propagation Letters, vol. 13, pp. 71-74, 2014.

[32] T. Björninen, J. Virkki, L. Sydanheimo, and L. Ukkonen, "Possibilities of 3D direct write dispensing for textile UHF RFID tag manufacturing," in Proceedings of the IEEE International Symposium on Antennas and Propagation \& USNC/URSI National Radio Science Meeting, pp. 1316-1317, IEEE, Vancouver, Canada, June 2015.

[33] T. Björninen, J. Virkki, L. Sydanheimo, and L. Ukkonen, "Manufacturing of antennas for passive UHF RFID tags by direct write dispensing of copper and silver inks on textiles," in Proceedings of the International Conference on Electromagnetics in Advanced Applications (ICEAA '15), pp. 589-592, IEEE, Turin, Italy, September 2015.

[34] J. Lilja, P. Salonen, T. Kaija, and P. De Maagt, "Design and manufacturing of robust textile antennas for harsh environments," IEEE Transactions on Antennas and Propagation, vol. 60, no. 9, pp. 4130-4140, 2012.

[35] J. Virkki, T. Björninen, S. Merilampi, L. Sydänheimo, and L. Ukkonen, "The effects of recurrent stretching on the performance of electro-textile and screen-printed ultra-high-frequency radio-frequency identification tags," Textile Research Journal, vol. 85, no. 3, pp. 294-301, 2015.

[36] Q. Bai and R. Langley, "Crumpling of PIFA textile antenna," IEEE Transactions on Antennas and Propagation, vol. 60, no. 1, pp. 63-70, 2012.

[37] T. Kaufmann, I.-M. Fumeaux, and C. Fumeaux, "Comparison of fabric and embroidered dipole antennas," in Proceedings of the 7th European Conference on Antennas and Propagation (EuCAP '13), pp. 3252-3255, IEEE, Gothenburg, Sweden, April 2013.

[38] G. Paul, R. Torah, K. Yang, S. Beeby, and J. Tudor, "An investigation into the durability of screen-printed conductive tracks on textiles," Measurement Science and Technology, vol. 25, no. 2, Article ID 025006, 11 pages, 2014.

[39] C. Hertleer, A. Van Laere, H. Rogier, and L. Van Langenhove, "Influence of relative humidity on textile antenna performance," Textile Research Journal, vol. 80, no. 2, pp. 177-183, 2010.

[40] M. Toivonen, T. Björninen, L. Sydanheimo, L. Ukkonen, and Y. Rahmat-Samii, "Impact of moisture and washing on the 
performance of embroidered UHF RFID tags," IEEE Antennas and Wireless Propagation Letters, vol. 12, pp. 1590-1593, 2013.

[41] R. Salvado, C. Loss, R. Gonçalves, and P. Pinho, "Textile materials for the design of wearable antennas: a survey," Sensors, vol. 12, no. 11, pp. 15841-15857, 2012.

[42] Y. Y. Fu, Y. L. Chan, M. H. Yang et al., "Experimental study on the washing durability of electro-textile UHF RFID tags," IEEE Antennas and Wireless Propagation Letters, vol. 14, pp. 466-469, 2015.

[43] T. Kellomäki, J. Virkki, S. Merilampi, and L. Ukkonen, “Towards washable wearable antennas: a comparison of coating materials for screen-printed textile-based UHF RFID tags," International Journal of Antennas and Propagation, vol. 2012, Article ID 476570, 11 pages, 2012.

[44] M. L. Scarpello, I. Kazani, C. Hertleer, H. Rogier, and D. Vande Ginste, "Stability and efficiency of screen-printed wearable and washable antennas," IEEE Antennas and Wireless Propagation Letters, vol. 11, pp. 838-841, 2012.

[45] I. Kazani, M. L. Scarpello, C. Hertleer et al., "Washable screen printed textile antennas," Advances in Science and Technology, vol. 80, pp. 118-122, 2012.

[46] I. Kazani, F. Declercq, M. L. Scarpello et al., "Performance study of screen-printed textile antennas after repeated washing," Autex Research Journal, vol. 14, no. 2, pp. 47-54, 2014.

[47] Fujitsu WT-A522 UHF RFID Laundry Tag, November 2015, http://rfid.atlasrfidstore.com/hs-fs/hub/300870/file-1523101876pdf/Tech_Spec_Sheets/Misc./ATLAS_Fujitsu_Laundry_Tag.pdf.

[48] TAGSYS' LinTRAK UHF Tag, November 2015, http://www.tagsysrfid.com/var/uploads/documents/54ddbad6c7d767a5f67158cce92dba92b6ce26f972f27.pdf.

[49] Less EMF Pure Copper Polyester Taffeta Fabric, http://www.lessemf.com/1212.pdf.

[50] NXP UCODE G2iL IC, http://www.nxp.com/documents/data_ sheet/SL3S1203_1213.pdf.

[51] ESL 243 White epoxy coating, http://www.electroscience.com/ pdf/243-White.pdf.

[52] Voyantic Ltd., Espoo, Finland, http://www.voyantic.com/.

[53] X DuPont, PE772 stretchable encapsulant datasheet, November 2015, http://www.dupont.com/content/dam/assets/productsand-services/electronic-electrical-materials/assets/datasheets/ prodlib/PE772.pdf.

[54] ISO 6330: Textiles, "Domestic washing and drying procedures for textile testing," 2012. 

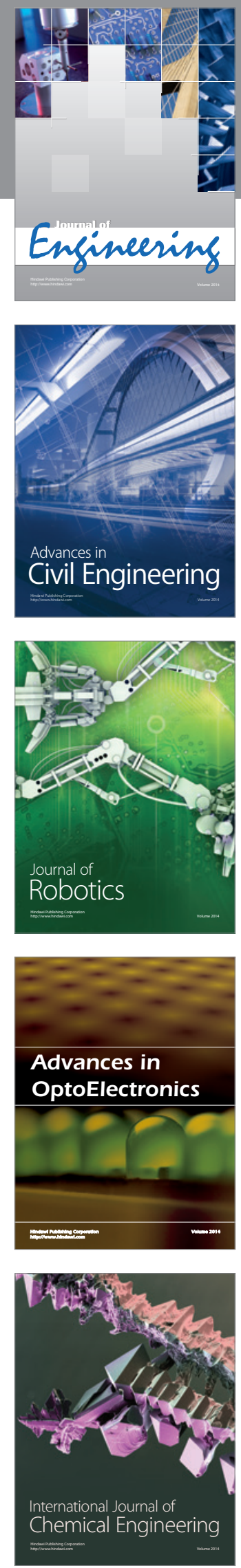

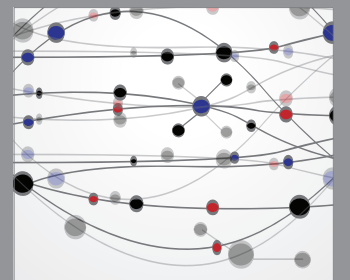

The Scientific World Journal
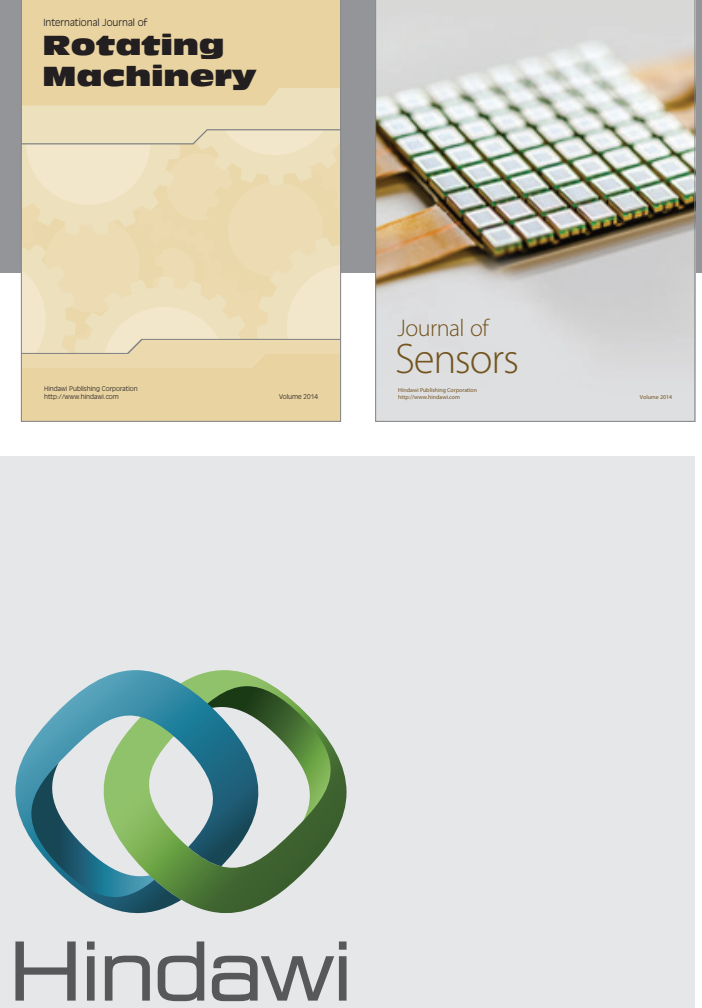

Submit your manuscripts at http://www.hindawi.com
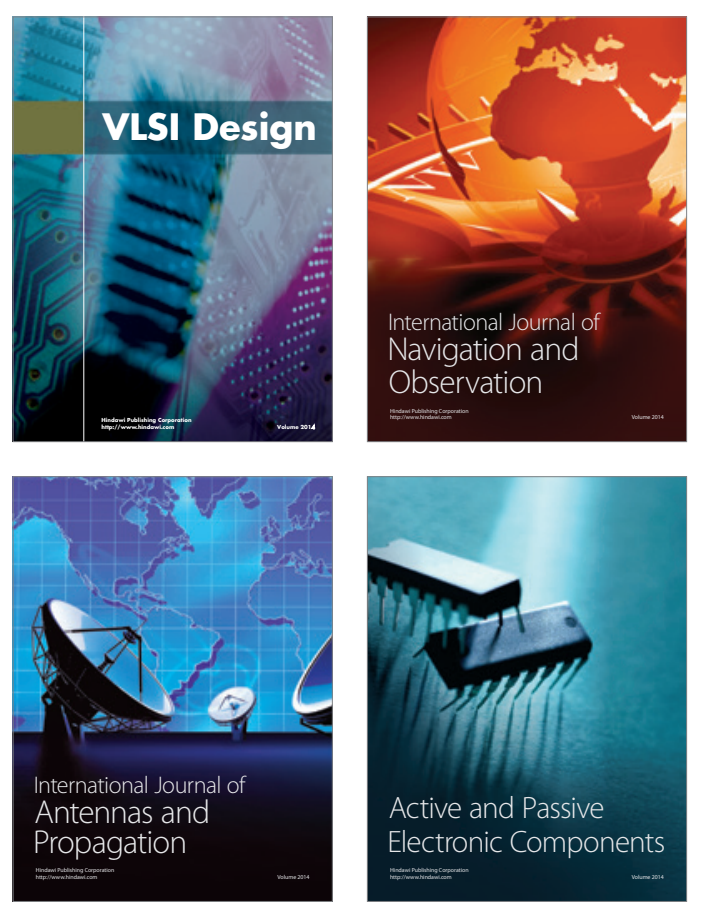
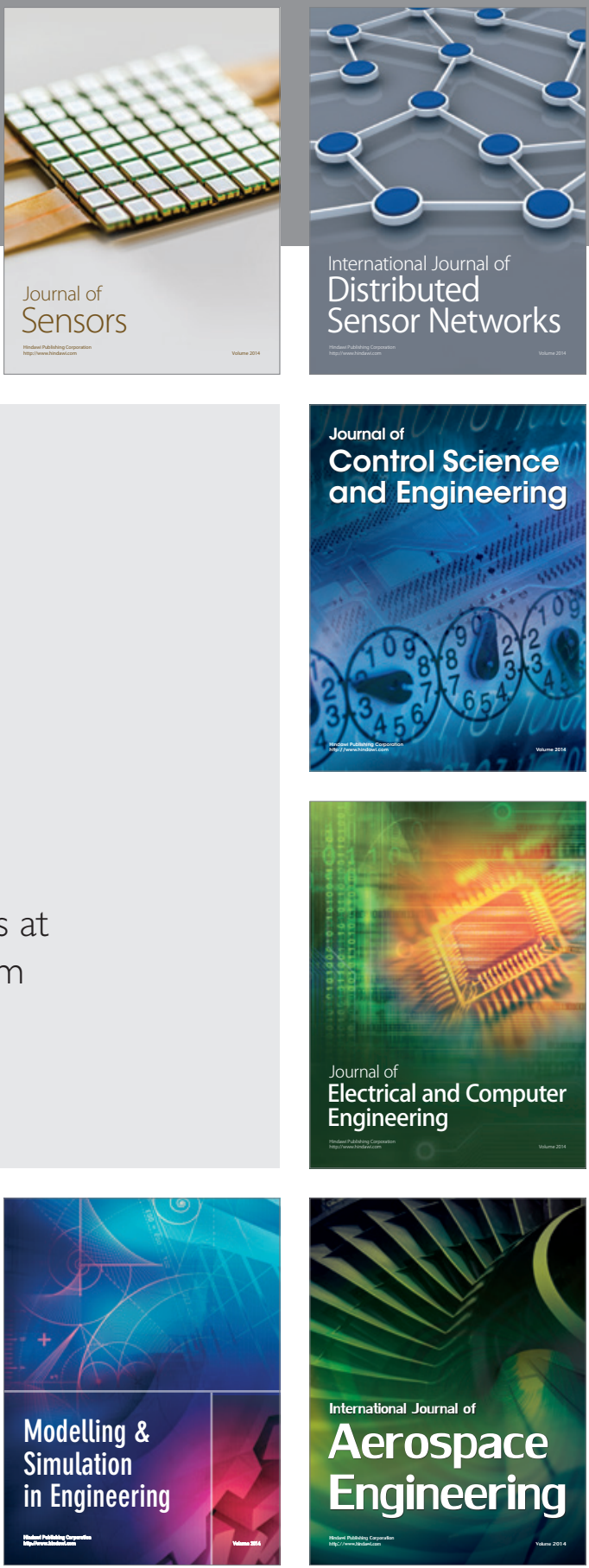

Journal of

Control Science

and Engineering
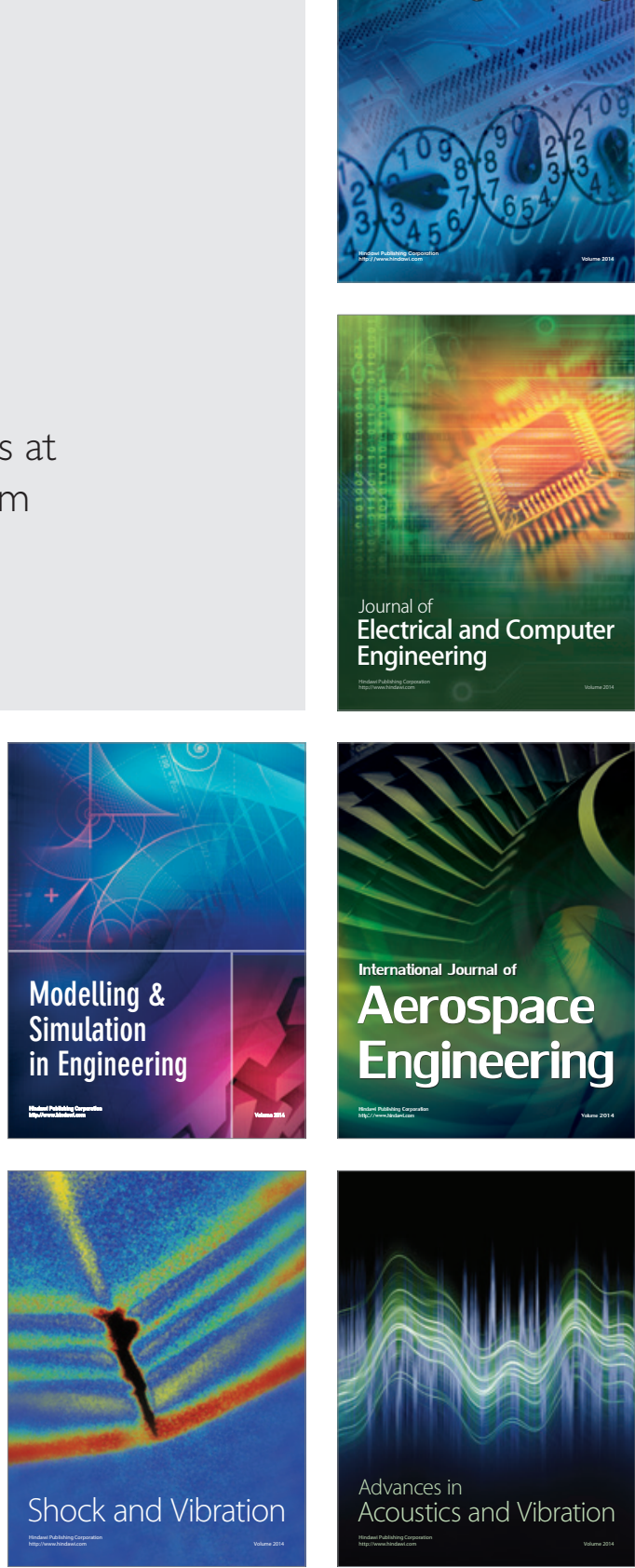